\title{
Parameterization of All Multivariable Deadbeat Tracking Controllers ${ }^{\dagger}$
}

\author{
Manabu Yamada* and Yasuyuki FunAhashr**
}

\begin{abstract}
This paper presents an explicit parameterization of all deadbeat tracking controllers for multi-input multioutput (MIMO) control systems in one-degree of freedom compensation scheme. The advantages over the previous results are as follows: Firstly, both all deadbeat tracking controllers and all achievable deadbeat responses are characterized by completely free parameters in an explicit form. Moreover, the relationship between the number of the settling step and the free parameters is made clear. Secondly, the class of the reference inputs for which deadbeat tracking is achieved is generalized. Thirdly, the design method is obtained not only for the case that the plant matrix is square but also for the non-square case. Fourthly, the design algorithm consists of simple polynomial matrix equations and the reduction to Hermite form over polynomials, while the previous algorithms in one-degree of freedom compensation scheme involve some troublesome calculations, e.g., the reduction to Smith-McMillan form of rational functions.
\end{abstract}

Key Words : multi-input multi-output (MIMO) system, discrete-time system, deadbeat tracking control, parameterization

\section{Introduction}

This paper is concerned with a deadbeat tracking control for discrete-time systems. In a deadbeat tracking control system, the controlled output can follow exactly a reference input in a finite time. However, it has been widely accepted that a deadbeat control has some troublesome problems from the practical point of view $^{8), 14)}$, for example, an excessive deterioration of tracking performance against disturbances or plant perturbations and so on.

In recent years, for single-input single-output (SISO) control systems, many effective design methods of deadbeat controllers have been studied to overcome such drawbacks, for example, deadbeat controllers with optimal robustness ${ }^{2), 17), 19)}$, ones with optimal robust performance ${ }^{15), 16)}$, ones with both optimal rejection for disturbances and optimal transient response for a step reference input $^{6), 12)}$ and so ${ }^{4}{ }^{4}$. For multi-input multi-output (MIMO) control systems, Zhao and Kimura ${ }^{18)}$ has presented a design method of deadbeat controllers taking into account the robustness of the systems in one-degree of

$\dagger$ Partially presented at 27th SICE Symposium on Control Theory (1998 • 5)

* Research Center for Micro-Structure Devices, Nagoya Institute of Technology, Nagoya

** Department of Mechanical Engineering, Nagoya Institute of Technology, Nagoya

(Received March 29, 2002)

(Revised November 8, 2002) freedom compensation scheme. This controller can minimize a robustness index, which is essentially the same as $L_{2}-$ norm of the sensitivity function. The reference input is restricted to a step signal. In 20), the result of 18 ) has been extended to two-degree of freedom compensation scheme. In 13), a design method based on geometric approach has been presented. However, these design algorithms of MIMO deadbeat controllers involve some troublesome calculations, e.g., the reduction to Smith-McMillan form of rational functions.' In 3), by using Hermite form over $R H_{\infty}$ instead of Smith-McMillan form, a simple design method of MIMO deadbeat controllers in two-degree of freedom compensation scheme has been proposed.

On the other hand, Nobuyama ${ }^{11}$ has proposed an explicit parameterization of all deadbeat controllers for a step reference input. The parameterization not only can make clear the characterization of both all deadbeat tracking controllers and all achievable deadbeat responses but also can make it easy to solve deadbeat problems to improve the drawbacks. In 1), the class of the reference inputs for which deadbeat tracking is achieved has been generalized. However, these results on parameterization are restricted to SISO control systems.

This paper presents an explicit parameterization of all deadbeat tracking controllers for MIMO control systems in one-degree of freedom compensation scheme. The advantages over the previous results are as follows: Firstly, both all deadbeat tracking controllers and all achievable deadbeat responses are characterized by com- 
pletely free parameters in an explicit form. Moreover the relationship between the number of the settling step and the free parameters is made clear. Secondly, the class of the reference inputs for which deadbeat tracking is achieved is generalized. Thirdly, the design method is obtained not only for the case that the plant matrix is square but also for the non-square case. Finally, the design algorithm consists of simple polynomial matrix equations and the reduction to Hermite form over polynomials, while the previous algorithms in one-degree of freedom compensation scheme involve some troublesome calculations, e.g., the reduction to Smith-McMillan form of rational functions. In a numerical example, it is demonstrated that the proposed parameterization makes it easy to find the optimal deadbeat response and to solve the optimal controller minimizing the maximum value of the controlled error in transient response.

The following notations are used throughout this paper.

$Z_{+}$: Set of all nonnegative integers

$R H_{\infty}^{m \times n}$ : Set of all $m \times n$ matrices whose elements are stable real rational functions

$R\left[z^{-1}\right]^{m \times n}:$ Set of $m \times n$ matrices whose elements are polynomials in the one-delay shift operator of $z^{-1}$ with real coefficients

For an $H(z) \in R\left[z^{-1}\right]^{m \times n}$, deg $H$ denotes the degree of $H(z)$ in $z^{-1}$. Let $A_{1}(z), A_{2}(z) \in R\left[z^{-1}\right]^{m \times m}$. If $A_{1}(z)=$ $U(z) A_{2}(z) V(z)$, where $U(z)$ and $V(z)$ are unimodular matrices, then $A_{1}(z)$ and $A_{2}(z)$ are equivalent, which is denoted by $A_{1}(z) \sim A_{2}(z)^{10)}$.

\section{Preliminaries}

\section{1 Problem Formulation}

Consider a multi-input multi-output discrete-time feedback control system as shown in Fig. 1. $P(z)$ is the linear time-invariant plant with $n$-inputs and $m$-outputs. It is assumed that the number of the inputs is no less than that of the outputs, i.e., $n \geq m$ and $\operatorname{rank} P(z)=m . C(z)$ is the linear time-invariant controller to be designed. For the sake of notation simplicity, we shall omit the indeterminate " $z$ " in $z$ transform of signal sequences and transfer functions throughout this paper. $r$ is the reference input to be tracked by the controlled output $y$. The reference input is generated by a linear time-invariant system and is

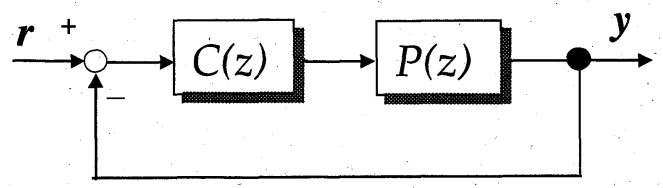

Fig. 1 MIMO discrete time control system described as follows :

$$
r=\tilde{D}_{r}^{-1} n_{r}
$$

where $\tilde{D}_{r} \in R\left[z^{-1}\right]^{m \times m}$ is given and $n_{r} \in R\left[z^{-1}\right]^{m}$ is arbitrary. $e(:=r-y)$ is the controlled error.

The coprime factorizations of $P$ over polynomials in $z^{-1}$ can be written as

$$
P=N D^{-1}=\widetilde{D}^{-1} \tilde{N}
$$

where $N, \tilde{N} \in R\left[z^{-1}\right]^{m \times n}, D \in R\left[z^{-1}\right]^{n \times n}$ and $\tilde{D} \in R\left[z^{-1}\right]^{m \times m}$. There exist $X_{0}, \tilde{X}_{0} \in R\left[z^{-1}\right]^{n \times m}, \quad Y_{0} \in R\left[z^{-1}\right]^{n \times n}$ and $\tilde{Y}_{0} \in$ $R\left[z^{-1}\right]^{m \times m}$ such that

$$
\left[\begin{array}{cc}
Y_{0} & X_{0} \\
-\tilde{N} & \tilde{D}
\end{array}\right]\left[\begin{array}{cc}
D & -\tilde{X}_{0} \\
N & \tilde{Y}_{0}
\end{array}\right]=\left[\begin{array}{cc}
D & -\tilde{X}_{0} \\
N & \tilde{Y}_{0}
\end{array}\right]\left[\begin{array}{cc}
Y_{0} & X_{0} \\
-\tilde{N} & \tilde{D}
\end{array}\right]=I_{m+n}
$$

All stabilizing controllers of $P$ are given by

$$
C=\left(\tilde{X}_{0}+D Q\right)\left(\tilde{Y}_{0}-N Q\right)^{-1},
$$

where $Q \in R H_{\infty}^{n \times m}$ is arbitrary. According to 11) and 14), a deadbeat tracking controller is defined as follows.

\section{Definition 1}

A controller of Eq. (2.4) is called deadbeat tracking controller if the controlled error for the reference input $r$ of Eq. (2.1) settles down to exactly zero in a finite number of control step, or equivalently $e \in R\left[z^{-1}\right]^{m}$. Then the degree of $e$ is called the settling step.

The main objectives of this paper are not only to make clear the necessary and sufficient condition for the existence of a deadbeat tracking controller but also to present an explicit parameterization of all deadbeat tracking controllers.

\subsection{Reduction to Hermite form}

Let the column Hermite form ${ }^{7}$ (or column echelon form $^{9)}$ ) over $R\left[z^{-1}\right]$ of $N$ of Eq. (2.2) be

$$
N=\widehat{N} U_{N} \text {, }
$$

where $\widehat{N}$ is a lower triangular matrix, which is denoted by

$$
\begin{aligned}
& \widehat{N}=\left[\widehat{n}_{i j}\right] \in R\left[z^{-1}\right]^{m \times n}, \\
& \widehat{n}_{i j}=0, i<j,
\end{aligned}
$$

and $U_{N} \in R\left[z^{-1}\right]^{n \times n}$ is a unimodular matrix ${ }^{10)}$. This reduction can be obtained easily by simple elementary column operations $^{7), 9)} . \quad \hat{n}_{i i} \in R\left[z^{-1}\right], i=1 \cdots m$ are decomposed as

$$
\widehat{n}_{i i}=\widehat{n}_{i i}^{u} \widehat{n}_{i i}^{s},
$$

where $\hat{n}_{i i}^{s} \in R\left[z^{-1}\right], \quad i=1 \cdots m$ contain all asymptotically stable zeros and $\hat{n}_{i i}^{u} \in R\left[z^{-1}\right], i=1 \cdots m$ contain the remaining zeros. Then $\hat{N}$ can be expressed as

$$
\widehat{N}=\widehat{N}^{u} \widehat{N}^{s}
$$

where

$$
\begin{aligned}
& \widehat{N}^{u}=\widehat{N}_{1}^{u}\left[I_{m} \quad 0\right] \in R\left[z^{-1}\right]^{m \times n}, \\
& \widehat{N}_{1}^{u}=\left[\begin{array}{ccc}
\widehat{n}_{11}^{u} & & 0 \\
\vdots & \ddots & \\
\widehat{n}_{m 1}^{u} & \cdots & \widehat{n}_{m m}^{u}
\end{array}\right] \in R\left[z^{-1}\right]^{m \times m},
\end{aligned}
$$




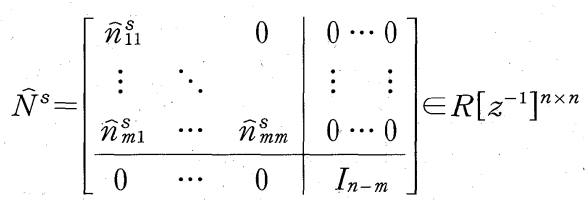

and $\hat{n}_{i j}^{u}$ and $\hat{n}_{i j}^{s}, i>j$ can be obtained easily by solving some simple Diophantine equations for given $\widehat{n}_{i i}^{u}$ and $\widehat{n}_{i i}^{s}$, $i=1 \cdots m$ of Eq. (2.6). See Appendix A for detail.

Similarly, let the row Hermite form ${ }^{7}$ ) (or row echelon form ${ }^{9)}$ ) over $R\left[z^{-1}\right]$ of $\tilde{D}$ of Eq. (2.2) be

$$
\tilde{D}=U_{\tilde{D}} \hat{D} \text {, }
$$

where $\widehat{D}$ is an upper triangular matrix, which is denoted by

$$
\begin{aligned}
& \widehat{D}=\left[\widehat{d}_{i j}\right] \in R\left[z^{-1}\right]^{m \times m}, \\
& \widehat{d}_{i j}=0, i>j,
\end{aligned}
$$

and $U_{\tilde{D}} \in R\left[z^{-1}\right]^{m \times m}$ is a unimodular matrix ${ }^{10)} . \quad \widehat{d}_{i i} \in$ $R\left[z^{-1}\right], i=1 \cdots m$ are decomposed as

$$
\hat{d}_{i i}=\bar{d}_{i i}^{u} \widehat{d}_{i i}^{s}
$$

where $\widehat{d}_{i i}^{s} \in R\left[z^{-1}\right], \quad i=1 \cdots m$ contain all asymptotically stable zeros and $\hat{d}_{i i}^{u} \in R\left[z^{-1}\right], i=1 \cdots m$ contain the remaining zeros. Then $\hat{D}$ can be expressed as

$$
\widehat{D}=\widehat{D}^{s} \widehat{D}^{u},
$$

where

$$
\begin{aligned}
& \widehat{D}^{s}=\left[\begin{array}{ccc}
\hat{d}_{11}^{s} & \cdots & \widehat{d}_{1 m}^{s} \\
& \ddots & \vdots \\
0 & & \hat{d}_{m m}^{s}
\end{array}\right] \in R\left[z^{-1}\right]^{m \times m}, \\
& \widehat{D}^{u}=\left[\begin{array}{ccc}
\hat{d}_{11}^{u} & \cdots & \hat{d}_{1 m}^{u} \\
& \ddots & \vdots \\
0 & \ddots & \widehat{d}_{m m}^{u}
\end{array}\right] \in R\left[z^{-1}\right]^{m \times m}
\end{aligned}
$$

and $\widehat{d}_{i j}^{s}$ and $\hat{d}_{i j}^{u}, i<j$ can be obtained easily by solving some simple Diophantine equations for given $\hat{d}_{i i}^{u}$ and $\hat{d}_{i i}^{s}$, $i=1 \cdots m$ of Eq. (2.10) as similar to Eq. (2.7).

\section{Main Results}

From Eqs. (2.2) and (2.4),

$$
\begin{aligned}
e & =\left(I_{m}+P C\right)^{-1} r \\
& =\left(\tilde{Y}_{0}-N Q\right) \tilde{D} \tilde{D}_{r}^{-1} n_{r} \\
& =E n_{r},
\end{aligned}
$$

where $E$ is given by

$$
E=\left(\tilde{Y}_{0}-N Q\right) \tilde{D} \widetilde{D}_{r}^{-1} \text {. }
$$

The deadbeat condition means that $e \in R\left[z^{-1}\right]^{m}$ for any $n_{r} \in R\left[z^{-1}\right]^{m}$, which is equivalent to that $E=R\left[z^{-1}\right]^{m \times m}$. Equation (3.2) can be represented as follows:

$$
N Q \tilde{D}+E \tilde{D}_{r}=\tilde{Y}_{0} \tilde{D} .
$$

Using the Hermite form of $N$ and $\tilde{D}$, the following lemma is obtained.

\section{Lemma 1}

The next three conditions are equivalent.

(i) There exists a deadbeat tracking controller.

(ii) There exist $Q \in R H_{\infty}^{n \times m}$ and $E \in R\left[z^{-1}\right]^{m \times m}$ satisfy- ing Eq. (3.3) .

(iii) There exist $\bar{Q} \in R\left[z^{-1}\right]^{m \times m}$ and $E \in R\left[z^{-1}\right]^{m \times m}$ satisfying

$\widehat{N}_{1}^{u} \bar{Q} \widehat{D}^{u}+E \widetilde{D}_{r}=\tilde{Y}_{0} \tilde{D}$.

\section{Proof :}

(i ) $\Leftrightarrow($ ii ) : Obvious.

(ii) $\Leftarrow\left(\right.$ iii ) : Suppose that there exist $\bar{Q} \in R\left[z^{-1}\right]^{m \times m}$ and $E$ $\in R\left[z^{-1}\right]^{m \times m}$ satisfying Eq. (3.4). Let

$$
Q=\left(\widehat{N}^{s} U_{N}\right)^{-1}\left[\begin{array}{l}
\bar{Q} \\
\bar{W}
\end{array}\right]\left(U_{\tilde{D}} \widehat{D}^{s}\right)^{-1},
$$

where $\bar{W} \in R H_{\infty}^{(n-m) \times m}$. Since $\left(\widehat{N}^{s}\right)^{-1} \in R H_{\infty}^{n \times n}$ and $\left(\widehat{D}^{s}\right)^{-1} \in$ $R H_{\infty}^{m \times m}$, this $Q$ is an element of $R H_{\infty}^{n \times m}$. Moreover, from Eq. (3.4), Equation (3.3) is satisfied. Accordingly, Condition (ii) holds.

(ii) $\Rightarrow$ (iii) : Suppose that there exist $Q \in R H_{\infty}^{n \times m}$ and $E \in$ $R\left[z^{-1}\right]^{m \times m}$ satisfying Eq. (3.3). Let

$$
\bar{Q}=\left[\begin{array}{ll}
I_{m} & 0
\end{array}\right] \widehat{N}^{s} U_{N} Q U_{\tilde{D}} \widehat{D}^{s} .
$$

Then, from Eq. (3.3), Equation (3.4) is satisfied. The remainings of the proof are to show that $\bar{Q} \in R\left[z^{-1}\right]^{m \times m}$. Since $Q \in R H_{\infty}^{n \times m}$, it follows that $\bar{Q} \in R H_{\infty}^{n \times m}$. Note that since $\widetilde{Y}_{0} \widetilde{D}-E \widetilde{D}_{r}$ is a polynomial matrix in $z^{-1}, \widehat{N}_{1}^{u} \bar{Q} \widehat{D}^{u} \in$ $R\left[z^{-1}\right]^{m \times m}$ from Eq. (3.4). Since all zeros of both $\widehat{N}_{1}^{u}$ and $\widehat{D}^{u}$ are unstable, $\bar{Q}$ is an element of $R\left[z^{-1}\right]^{m \times m}$. Accordingly, Condition (iii) holds.

Q.E. D.

\section{Remark :}

While Equation (3.3) includes a rational function, Equation (3.4) consists of polynomials. Accordingly, by Condition (iii), the problem of finding a deadbeat tracking controller is reduced to that of finding the solution of the polynomial matrix equation of Eq. (3.4). As shown below, this reduction plays an important role on obtaining the necessary and sufficient condition for the existence of a deadbeat tracking controller and the parameterization of all deadbeat tracking controllers.

From Eqs. (2.8) and (2.11), Equation (3.4) can be represented as follows:

$$
E=-\left(\widehat{N}_{1}^{u} \bar{Q}-\widetilde{Y}_{0} U_{\tilde{D}} \widehat{D}^{s}\right) \widehat{D}^{u} \widehat{D}_{r}^{-1} .
$$

Define the left coprime factorization of $-\widehat{D}^{u} \widetilde{D}_{r}^{-1}$ over polynomials in $z^{-1}$ as

$$
\left(X^{*}\right)^{-1} \tilde{X}^{*}=-\widehat{D}^{u} \tilde{D}_{r}^{-1},
$$

where $X^{*} \in R\left[z^{-1}\right]^{m \times m}$ and $\tilde{X}^{*} \in R\left[z^{-1}\right]^{m \times m}$ are left coprime. Then

$$
E=\left(\widehat{N}_{1}^{u} \bar{Q}-\tilde{Y}_{0} U_{\tilde{D}} \widehat{D}^{s}\right)\left(X^{*}\right)^{-1} \tilde{X}^{*} .
$$

The following theorem provides the necessary and sufficient condition for the existence of a deadbeat tracking controller.

\section{Theorem 1}

The next three conditions are equivalent.

(i ) There exists a deadbeat tracking controller. 
(ii) There exist $F \in R\left[z^{-1}\right]^{m \times m}$ and $\bar{Q} \in R\left[z^{-1}\right]^{m \times m}$ such that

$$
\widehat{N}_{1}^{u} \bar{Q}-F X^{*}=\tilde{Y}_{0} U_{\tilde{D}} \widehat{D}^{s} .
$$

(iii)

$$
\left[\begin{array}{cc}
\widehat{N}_{1}^{u} & \tilde{Y}_{0} U_{\tilde{D}} \widehat{D}^{s} \\
0 & -X^{*}
\end{array}\right] \sim\left[\begin{array}{cc}
\widehat{N}_{1}^{u} & 0 \\
0 & -X^{*}
\end{array}\right] .
$$

Then $E \in R\left[z^{-1}\right]^{m \times m}$ is given by

$$
E=F \tilde{X}^{*} \text {. }
$$

Proof :

From Lemma 1 and Eq. (3.9), there exists a deadbeat tracking controller if and only if there exist $\bar{Q} \in R\left[z^{-1}\right]^{m \times m}$ and $E \in R\left[z^{-1}\right]^{m \times m}$ satisfying Eq. (3.9). Since $X^{*} \in$ $R\left[z^{-1}\right]^{m \times m}$ and $\tilde{X}^{*} \in R\left[z^{-1}\right]^{m \times m}$ are left coprime, by Lemma $\mathrm{B} 1$ in Appendix B, equivalently, there exist $\bar{Q} \in R\left[z^{-1}\right]^{m \times m}$ and $F \in R\left[z^{-1}\right]^{m \times m}$ satisfying $F=\left(\widehat{N}_{1}^{u} \bar{Q}-\tilde{Y}_{0} U_{\tilde{D}} \widehat{D}^{s}\right)\left(X^{*}\right)^{-1}$. Notice that this equation is the same as Eq. (3.10). Consequently, Conditions ( i ) and (ii) are equivalent and we obtain Eq. (3.12). Moreover, it is straightforward that Condition (ii) is equivalent to Condition (iii) ${ }^{10)}$. The proof is completed.

Q.E.D.

\section{Remark :}

This theorem states that the problem of finding a deadbeat tracking controller is reduced to that of finding the solution of the simple polynomial matrix equation of Eq. (3.10). The following corollary provides simpler and more useful expression of existence condition of a deadbeat tracking controller.

\section{Corollary 1}

There exists a deadbeat tracking controller, if $\widehat{N}_{1}^{u}$ and $\alpha_{X} I_{m}$ are left coprime, where $\alpha_{X} \in R\left[z^{-1}\right]$ is the largest invariant polynomial of $X^{*} \in R\left[z^{-1}\right]^{m \times m}$.

\section{Proof :}

Suppose that $\widehat{N}_{1}^{u}$ and $\alpha_{X} I_{m}$ are left coprime. Then there exist $\bar{Q}_{0} \in R\left[z^{-1}\right]^{m \times m}$ and $F_{0} \in R\left[z^{-1}\right]^{n \times m}$ such that

$$
\widehat{N}_{1}^{u} \bar{Q}_{0}+\alpha_{X} F_{0}=\tilde{Y}_{0} U_{\tilde{D}} \widehat{D}^{s} .
$$

Since $\alpha_{X}\left(X^{*}\right)^{-1}$ is a polynomial matrix in $z^{-1}$, both $\bar{Q}=$ $\bar{Q}_{0}$ and $F=-\alpha_{X} F_{0}\left(X^{*}\right)^{-1}$ are polynomial matrices in $z^{-1}$ and are the solutions of Eq. (3.10). The proof is completed.

Q.E. D.

The following theorem presents an explicit parameterization of all deadbeat tracking controllers and makes the relationship between the number of the settling step and the degree of free polynomial matrix in the parameterization clear.

\section{Theorem 2}

Assume that $\widehat{N}_{1}^{u}$ and $\alpha_{x} I_{m}$ are left coprime. Let $\bar{Q}^{*} \in$ $R\left[z^{-1}\right]^{m \times m}$ and $F^{*} \in R\left[z^{-1}\right]^{m \times m}$ be the minimum degree solution $\bar{Q}$ and $F$ with respect to $F$ of Eq. (3.10), respectively. Then all deadbeat tracking controllers can be represented as Eq. (2.4) with

$$
Q=\left(\widehat{N}^{s} U_{N}\right)^{-1}\left[\begin{array}{c}
\bar{Q}^{*}+W X^{*} \\
\bar{W}
\end{array}\right]\left(U_{\tilde{D}} \widehat{D}^{s}\right)^{-1} \in R H_{\infty}^{n \times m},
$$

where both $W \in R\left[z^{-1}\right]^{m \times m}$ and $\bar{W} \in R H_{\infty}^{(n-m) \times m}$ are arbitrary. With this controller, the controlled error sequence is given by

$$
e=\left(F^{*}+\widehat{N}_{1}^{u} W\right) \tilde{X}^{*} n_{r} \in R\left[z^{-1}\right]^{m} .
$$

Moreover, in terms of the settling step, the following properties hold.

(i) For $W=0$, the minimum settling step deadbeat tracking controller is obtained. The number of the settling step is given by $\operatorname{deg} F^{*} \tilde{X}^{*} n_{r}$.

(ii) For a given $W \neq 0$, the number of the settling step is not more than deg $\widehat{N}_{1}^{u}+\operatorname{deg} W+\operatorname{deg} \tilde{X}^{*} n_{r}$.

\section{Proof :}

According to 10), the general solution of the bilateral polynomial matrix equation of Eq. (3.10) is given by

$$
\begin{aligned}
& \bar{Q}=\bar{Q}^{*}+W X^{*}, \\
& F=F^{*}+\widehat{N}_{1}^{u} W,
\end{aligned}
$$

where $W \in R\left[z^{-1}\right]^{m \times m}$ is arbitrary. Substituting Eq. (3.16) into Eqs. (3.5) and (3.12), Eqs. (3.14) and (3.15) are obtained, respectively. Moreover the minimum degree solution of $F^{*}$ has the following property:

$$
\operatorname{deg} F^{*}<\operatorname{deg} \hat{N}_{1}^{u} \text {. }
$$

Therefore, the properties ( i ) and (ii) are obtained. The proof is completed.

Q.E. D.

\section{Remark 1:}

In 10), we can find both how to solve the minimum degree solution of $\bar{Q}^{*}$ and $F^{*}$ and the proof of the uniqueness of the solution.

\section{Remark 2 :}

In this theorem, the parameterization of all deadbeat tracking controllers is characterized by completely free $W$ $\in R\left[z^{-1}\right]^{m \times m}$ and $\bar{W} \in R H_{\infty}^{(n-m) \times m}$ in an explicit form. Moreover, the number of the settling step, denoted by $N_{\text {set }}$ $\in Z_{+}$, can be set arbitrarily by an appropriate choice of the degree of $W \in R\left[z^{-1}\right]^{m \times m}$ under

$$
N_{\text {set }} \geq \operatorname{deg} F^{*} \tilde{X}^{*} n_{r},
$$

and it is independent of $\bar{W} \in R H_{\infty}^{(n-m) \times m}$. In other words, the class of deadbeat tracking controllers with a given settling step, $N_{\text {set }} \in Z_{+}$, can be given by Eq. (3.14), where and both $W \in R\left[z^{-1}\right]^{m \times m}$ and $\bar{W} \in R H_{\infty}^{(n-m) \times m}$ are arbitrary under the degree constraint of

$$
\operatorname{deg} W \leq N_{\text {set }}-\operatorname{deg} \widehat{N}_{1}^{u}-\operatorname{deg} \tilde{X}^{*} n_{r} .
$$

Finally, we discuss the case that $\tilde{D}_{r}=d_{r} I_{m} \in R\left[z^{-1}\right]^{m \times m}$, where $d_{r} \in R\left[z^{-1}\right]$ is a given scalar polynomial in $z^{-1}$. Then we have the following corollary.

\section{Corollary 2}

There exists a deadbeat tracking controller if and only 
if $\widehat{N}_{1}^{u}$ and $d_{r} I_{m}$ are left coprime. Then all deadbeat tracking controllers can be represented as Eq. (2.4) and Eq. (3.14), where both $W \in R\left[z^{-1}\right]^{m \times m}$ and $\bar{W} \in R H_{\infty}^{(n-m) \times m}$ are arbitrary. With this controller, the controlled error sequence is given by Eq. (3.15). Moreover, the properties (i) and (ii) in Theorem 2 hold.

\section{Proof :}

Firstly, we will prove the part of "if". From the definition of $\alpha_{X} \in R\left[z^{-1}\right], \alpha_{X}$ divides $d_{r}$ over $R\left[z^{-1}\right]$. If $\widehat{N}_{1}^{u}$ and $d_{r} I_{m}$ are left coprime, by Corollary 1 , there exists a deadbeat tracking controller. Moreover we obtain the parameterization of Eq. (3.14) from Theorem 2. Secondly, we will prove the part of "only if". Suppose that there exists a deadbeat tracking controller, that is to say, there exist $F \in R\left[z^{-1}\right]^{m \times m}$ and $\bar{Q} \in R\left[z^{-1}\right]^{m \times m}$ satisfying Eq. (3.10). From Eq. (2.3), there exist $X_{0} \in R\left[z^{-1}\right]^{n \times m}$ and $\tilde{Y}_{0}$ $\in R\left[z^{-1}\right]^{m \times m}$ such that

$$
N X_{0}+\tilde{Y}_{0} \tilde{D}=I_{m} \text {. }
$$

Substituting Eqs. (2.5), (2.7), (2.8), (2.11) and (3.10), it follows that

$$
\widehat{N}_{1}^{u}\left(\left[\begin{array}{ll}
I_{m} & 0
\end{array}\right] \widehat{N}^{s} U_{N} X_{0}+\bar{Q} \widehat{D}^{u}\right)-F X^{*} \widehat{D}^{u}=I_{m} .
$$

From Eq.(3.8), we obtain

$$
\widehat{N}_{1}^{u}\left(\left[\begin{array}{ll}
I_{m} & 0
\end{array}\right] \widehat{N}^{s} U_{N} X_{0}+\bar{Q} \hat{D}^{u}\right)+d_{r} F \tilde{X}^{*}=I_{m} .
$$

This means that $\widehat{N}_{1}^{u}$ and $d_{r} I_{m}$ are left coprime. The proof is completed.

Q. E. D.

\section{Numerical example}

Consider the following 2-inputs 2-outputs proper discrete-time transfer function as a plant:

$$
P=\left[\begin{array}{cc}
\frac{0.44 z^{-1}\left(1+0.88 z^{-1}\right)}{\left(1-z^{-1}\right)\left(1-0.82 z^{-1}\right)} & \frac{0.88 z^{-1}\left(1+0.88 z^{-1}\right)}{\left(1-z^{-1}\right)\left(1-0.82 z^{-1}\right)} \\
\frac{0.86 z^{-1}\left(1-0.9 z^{-1}\right)}{\left(1-z^{-1}\right)\left(1-0.82 z^{-1}\right)} & \frac{0.95 z^{-1}\left(1-0.74 z^{-1}\right)}{\left(1-z^{-1}\right)\left(1-0.82 z^{-1}\right)}
\end{array}\right] .
$$

The reference input is given by

$$
r=\left[\begin{array}{cc}
1-z^{-1} & 0 \\
0 & \left(1-z^{-1}\right)^{2}
\end{array}\right]^{-1}\left[\begin{array}{c}
1 \\
0.1
\end{array}\right]
$$

First, we will demonstrate how to design the parameterization of all deadbeat tracking controllers by steps $1 \sim 3$ as shown below.

STEP 1: From section 2.2 and Appendix A, we obtain the following Hermite forms :

$$
\begin{aligned}
U_{N} & =\left[\begin{array}{ll}
1 & 2 \\
0 & 1
\end{array}\right], U_{\tilde{D}}=I_{2}, \\
\widehat{N}^{u} & =\left[\begin{array}{cc}
z^{-1} & 0 \\
0.195 z^{-1} & z^{-1}\left(1-1.097 z^{-1}\right)
\end{array}\right], \\
\widehat{N}^{s} & =\left[\begin{array}{cc}
0.44+0.387 z^{-1} & 0 \\
0.774 & -0.77
\end{array}\right],
\end{aligned}
$$

$$
\begin{aligned}
& \widehat{D}^{u}=\left[\begin{array}{cc}
\left(1-z^{-1}\right) & 0 \\
0 & \left(1-z^{-1}\right)
\end{array}\right], \\
& \widehat{D}^{s}=\left[\begin{array}{cc}
1-0.82 z^{-1} & 0 \\
0 & 1-0.82 z^{-1}
\end{array}\right] .
\end{aligned}
$$

STEP 2: From Eq. (3.8), we have

$$
X^{*}=\left[\begin{array}{cc}
1 & 0 \\
0 & 1-z^{-1}
\end{array}\right], \tilde{X}^{*}=-I_{2}, \alpha_{X}=1-z^{-1} .
$$

STEP 3 : Since $\widehat{N}^{u}$ and $\alpha_{X} I_{m}$ are left coprime, there exists a deadbeat tracking controller. From Theorem 2, the parameterization of all deadbeat tracking controllers is obtained explicitly by Eq. (2.4) with

$$
\begin{aligned}
Q= & \frac{1}{\left(1-0.82 z^{-1}\right)\left(1+0.880 z^{-1}\right)} \\
& \times\left[\begin{array}{cc}
6.82 & 2.60+2.29 z^{-1} \\
-2.28 & -1.30-1.14 z^{-1}
\end{array}\right] \\
& \times\left[\begin{array}{cc}
-0.153-0.547 z^{-1}+w_{11} & \left(1-z^{-1}\right) w_{12} \\
-6.97+w_{21} & -90.2+\left(1-z^{-1}\right) w_{22}
\end{array}\right],
\end{aligned}
$$

where $w_{i j} \in R\left[z^{-1}\right], i, j=1,2$ are arbitrary.

With this controller, the controlled error is represented explicitly as follows:

$$
\begin{aligned}
& {\left[\begin{array}{l}
e_{1} \\
e_{2}
\end{array}\right]=} \\
& \quad\left[\begin{array}{c}
1-z^{-1} \widehat{w}_{1} \\
-0.1-11.6 z^{-1}-0.195 z^{-1} \widehat{w}_{1}-z^{-1}\left(1-1.10 z^{-1}\right) \widehat{w}_{2}
\end{array}\right],
\end{aligned}
$$

where $\hat{w}_{i}=w_{i 1}-0.1 w_{i 2} \in R\left[z^{-1}\right], \quad i=1,2$, are arbitrary. Equation (4.4) shows that the deadbeat tracking response has the following properties.

(i ) All deadbeat controllers with minimum settling step are characterized by deg $\widehat{w}_{1}=0$ and $\widehat{w}_{2}=0$. Then all achievable minimum settling step deadbeat response is represented by

$$
\left[\begin{array}{l}
e_{1} \\
e_{2}
\end{array}\right]=\left[\begin{array}{c}
1-z^{-1} w_{10} \\
-0.1-11.6 z^{-1}-0.195 z^{-1} w_{10}
\end{array}\right], \hat{w}_{1}=w_{10} \in R .
$$

It is easy to find that the maximum value of the controlled error becomes

$$
\|e\|_{\infty}=\max \left\{1,\left|w_{10}\right|,\left|11.6+0.195 w_{10}\right|\right\} \text {. }
$$

Therefore the optimal solution of $w_{10} \in R$ minimizing the maximum value of the controlled error is obtained by $w_{10}=-9.71$ and then $\|e\|_{\infty}=9.71$. Fig. 2 shows the optimal deadbeat responses of the controlled errors under the minimum settling step.

(ii) If deg $\widehat{w}_{1} \geq 1$ or deg $\widehat{w}_{2} \geq 0$, then the number of the settling step is given by $\max \left(\operatorname{deg} \widehat{w}_{1}+1\right.$, deg $\left.\widehat{w}_{2}+2\right)$. For example, let

$$
\begin{aligned}
& \widehat{w}_{1}=w_{10}+w_{11} z^{-1} \in R\left[z^{-1}\right] \\
& \widehat{w}_{2}=w_{20} \in R .
\end{aligned}
$$




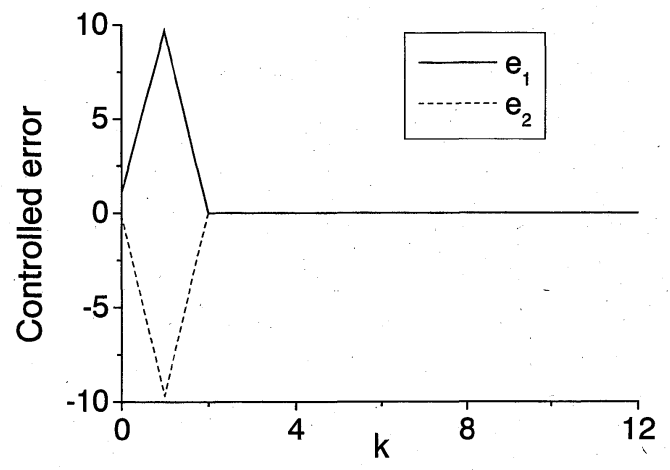

Fig. 2 Controlled errors for reference inputs (Case of the minimum settling step)

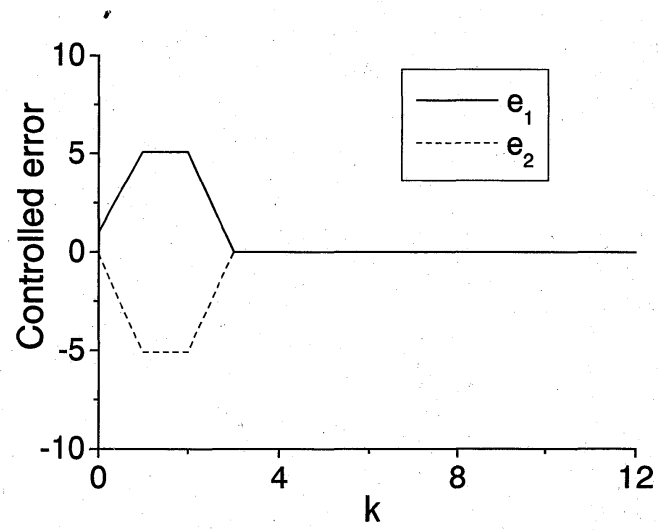

Fig. 3 Controlled errors for reference inputs (Case of 2 settling steps)

Then all achievable deadbeat responses under 2 settling steps are given by

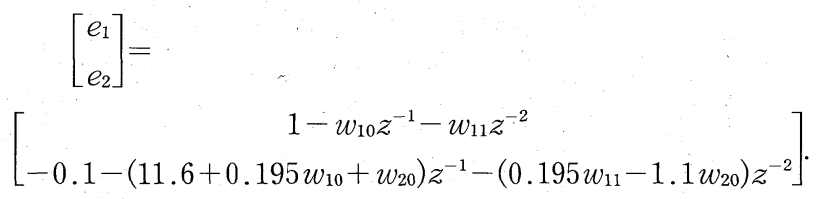

It is easy to find that the maximum value of the controlled error becomes

$$
\begin{aligned}
\|e\|_{\infty}= & \max \left\{1,\left|w_{10}\right|,\left|w_{11}\right|,\left|11.6+0.195 w_{10}+w_{20}\right|,\right. \\
& \left.\left|0.195 w_{11}-1.1 w_{20}\right|\right\} .
\end{aligned}
$$

Therefore the optimal solution minimizing the maximum value of the controlled error is obtained by $\left(w_{10}, w_{11}\right.$, $\left.w_{20}\right)=(-5.09,-5.09,-5.52)$ and then $\|e\|_{\infty}=5.09$. Fig. 3 shows the optimal deadbeat responses of the controlled errors under the settling step of $N_{\text {set }}=2$. In Figs. 2 and 3, we see that the controlled errors settle down to zero in the given settling control step. Moreover as increasing the settling steps, the maximum value of the controlled error becomes smaller.

\section{Conclusion}

This paper has presented parameterization of all MIMO deadbeat tracking controllers. The parameterization has been characterized by both a completely free polynomial matrix and a completely free rational function one in an explicit form. It has been shown that the settling step depends on the degree of the polynomial matrix. Moreover the relationship between the number of the settling step and the degree of the free polynomial matrix has been made clear.

\section{Acknowledgements}

The authors would like to thank the reviewers for their valuable comments.

\section{References}

1) A. Casavola, E. Mosca and P. Zecca: Robust ripple-free deadbeat control design, International Journal of Control, 72-6, 564/573 (1999)

2) Y. Funahashi and H. Kato: Minimal time dead-beat control with optimal robustness by two-parameter compensation scheme, Transactions of the Society of Instrument and Control Engineers, 24, 483/489 (1988) (in Japanese)

3) Y. Funahashi and H. Kato: Robust deadbeat control for MIMO systems, ibid, 27, 640/646 (1991). (in Japanese)

4) Y. Funahashi and H. Kato: Robust-tracking deadbeat control, International Journal of Control, 56, 213/225 (1992) •

5) M. J. Grimble and V. Kucera (Eds) : Polynomial Methods for Control Systems Design, Springer (1996)

6) K. Horiguchi, Nishimura, Osada and Tomita: Twodegree-of-freedom dead-beat control considering transient responses, Transactions of the Society of Instrument and Control Engineers, 25, 1046/1053 (1989) (in Japanese)

7) T. Kailath: Linear Systems, Prentice Hall (1980)

8) H. Kimura : Robust dead-beat control, Journal of the Society of Instrument and Control Engineers, 27-12, 1078/ 1084 (1988) (in Japanese)

9) S. Kodama and N. Suda: Matrix Theory for System Control, The Society of Instrument and Control Engineers (1978) (in Japanese)

10) V. Kucera: Discrete Linear Control: The Polynomial Equation Approach, Wiley (1979)

11) E. Nobuyama: Parametrization of all ripple-free deadbeat controllers, System and Control Letters, 21, 217/224 (1993)

12) T. Sugie and Y. Hata : Design of deadbeat control systems considering the maximum error bound, Transactions of the Society of Instrument and Control Engineers, 28, 931/938 (1992) (in Japanese)

13) S. Urikura and A. Nagata : Ripple-free deadbeat control for sampled-data systems, IEEE Transactions on Automatic Control, 32-6, 474/482 (1987)

14) M. Yamada: Finite-time Settling Control for Sampled Data, Journal of the Society of Instrument and Control Engineers, 38, 553/558 (1999). (in Japanese)

15) M. Yamada, Y. Funahashi and Z. Riadh: Design of deadbeat control system considering worst case steady-state tracking performance, Proceedings of the 36th IEEE: Conference on Decision and Control, San Diego, C. A, 351/356 (1997)

16) M. Yamada, Z. Riadh and Y. Funahashi : Deadbeat controI system with time-varying uncertainty: minimization of worst case steady-state tracking error, International Jour- 
nal of Control, 72-2, 141/149 (1999)

17) Y. Zhao and H. Kimura: Dead-beat control with robustness, International Journal of Control, 43, 1427/1440 (1986)

18) Y. Zhao and H. Kimura: Multivariable dead-beat control with robustness, ibid., 47, 229/235 (1988)

19) Y. Zhao and H. Kimura: Two-degree-of-freedom deadbeat control with robustness, ibid, 48, 303/315 (1988)

20) Y. Zhao and H. Kimura: Two-degree-of-freedom deadbeat control with robustness: multivariable case, ibid, 49 , 667/679 (1989)

\section{Appendix A}

In this appendix, we will show how to calculate $\widehat{N}^{u}$ and $\widehat{N}^{s}$ of Eq. (2.7) for a given $\widehat{N}$. For the sake of description simplicity, consider the case of $m=n=3$. Then Equation (2.7) is represented as

$$
\left[\begin{array}{ccc}
\hat{n}_{11} & 0 & 0 \\
\hat{n}_{21} & \widehat{n}_{22} & 0 \\
\hat{n}_{31} & \widehat{n}_{32} & \widehat{n}_{33}
\end{array}\right]=\left[\begin{array}{ccc}
\hat{n}_{11}^{u} & 0 & 0 \\
\hat{n}_{21}^{u} & \widehat{n}_{22}^{u} & 0 \\
\widehat{n}_{31}^{u} & \widehat{n}_{32}^{u} & \widehat{n}_{33}^{u}
\end{array}\right]\left[\begin{array}{ccc}
\hat{n}_{11}^{s} & 0 & 0 \\
\widehat{n}_{21}^{s} & \widehat{n}_{22}^{s} & 0 \\
\widehat{n}_{31}^{s} & \widehat{n}_{32}^{s} & \widehat{n}_{33}^{s}
\end{array}\right] .
$$

We can obtain $\hat{N}^{u}$ and $\hat{N}^{s}$ of Eq. $(2.7)$ for the given $\hat{N}$ by the following procedure.

Step 1: For given $\hat{n}_{i i}, i=1 \cdots 3$, we obtain $\widehat{n}_{i i}^{u}$ and $\hat{n}_{i i}^{s}, i=$ $1 \cdots 3$ from Eq. (2.6).

Step 2: From Eq. (A.1), we have the following Diophantine equations :

$$
\widehat{n}_{i+1, i}^{u} \widehat{n}_{i, i}^{s}+\widehat{n}_{i+1, i+1}^{u} \widehat{n}_{i+1, i}^{s}=\widehat{n}_{i+1, i}, i=1,2 .
$$

Since $\left(\bar{n}_{i, i}^{s}, \hat{n}_{i+1, i+1}^{u}\right), i=1,2$ are coprime, there exist the solutions of $\bar{n}_{i+1, i}^{u}, \bar{n}_{i+1, i}^{s}, i=1,2$ in Eq. (A.2). Hence we can obtain these solutions for given $\widehat{n}_{i+1, i}, i=1,2$.

Step 3: From Eq.(A.1), we have the following Diophantine equation :

$$
\widehat{n}_{31}^{u} \widehat{n}_{11}^{s}+\widehat{n}_{32}^{u} \widehat{n}_{21}^{s}+\widehat{n}_{33}^{u} \widehat{n}_{31}^{s}=\widehat{n}_{31} \text {. }
$$

Since $\left(\bar{n}_{11}^{s}, \bar{n}_{33}^{u}\right)$ are coprime, there exist the solution of $\hat{n}_{31}^{u}$ and $\hat{n}_{31}^{s}$ in Eq.(A.3). Hence we can obtain this solution for $\hat{n}_{32}^{u}$ and $\hat{n}_{21}^{s}$ obtained in step 2 and given $\hat{n}_{31}$. The procedure for obtaining $\bar{N}^{u}$ and $\widehat{N}^{s}$ of Eq. (A.1) is completed.

It is obvious that, for the general case of $m$ and $n$, we can obtain $\hat{N}^{u}$ and $\hat{N}^{s}$ of Eq. (2.7) for a given $\hat{N}$ by the similar procedure.

\section{Appendix B}

In this appendix, the lemma used in the proof of Theo- rem 1 is shown.

\section{Lemma B 1}

Let $A(z), B(z), C(z) \in R\left[z^{-1}\right]^{m \times m}$. Suppose that $B(z)$ and $C(z)$ are left coprime and $B(z)$ is nonsingular. Then the following statements are equivalent.
(a) $A(z) B(z)^{-1} C(z) \in R\left[z^{-1}\right]^{m \times m}$
(b). $A(z) B(z)^{-1} \in R\left[z^{-1}\right]^{m \times m}$

\section{Proof :}

$((\mathrm{a}) \Leftarrow(\mathrm{b}))$ : Obvious.

$((\mathrm{a}) \Rightarrow(\mathrm{b}))$ : Since $B(z)$ and $C(z)$ are left coprime, there exist $X_{2}(z), Y_{2}(z) \in R\left[z^{-1}\right]^{m \times m}$ satisfying

$$
B(z) X_{2}(z)+C(z) Y_{2}(z)=I_{m} \text {. }
$$

Then we have

$$
\begin{aligned}
A(z) B(z)^{-1} & =A(z) B(z)^{-1}\left(B(z) X_{2}(z)+C(z) Y_{2}(z)\right) \\
& =A(z) X_{2}(z)+A(z) B(z)^{-1} C(z) Y_{2}(z) .
\end{aligned}
$$

Consequently, if the statement of (a) holds, then the statement of (b) also holds.

Q.E. D.

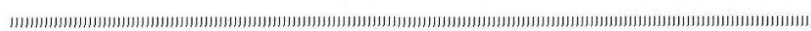

\section{Manabu YaMada (Member)}

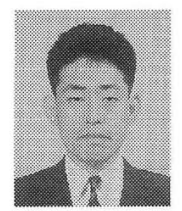

He received the B.E., M.E. and Dr. Eng. degrees from Nagoya Institute of Technology, JAPAN in 1988, 1990 and 1992, respectively. In 1992, He joined the Mechanical Engineering at Nagoya Institute of Technology as a Research Associate. Since 1999, he has been a Associate Professor of the Research Center for MicroStructure Devices at Nagoya Institute of Technology. His research interests are in the areas of digital control systems, robust control systems and optimal control systems. Dr. Yamada is a member of Japan Society of Mechanical Engineers.

\section{Yasuyuki Funahashi (Member)}

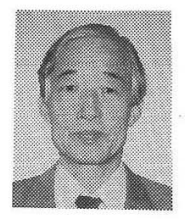

He received the Doctor's degree in Electrical Engineering from Nagoya University, JAPAN in 1968, and joined the Research Laboratory of Automatic Control in Nagoya University as a Research Associate. Since 1986, he has been a Professor of Mechanical Engineering at Nagoya Institute of Technology. His primary research interest is control theory. Prof. Funahashi is a member of IEEE and Japan Society of Mechanical Engineers. 